\title{
EFFECT OF SHELF CONDITIONS ON THE PHENOLIC FRACTION AND OXIDATION INDICES OF MONOVARIETAL EXTRA VIRGIN OLIVE OIL FROM CV. 'TAGGIASCA'
}

\author{
B. Lanza*, M.G. Di Serio, L. Giansante, G. Di Loreto and L. Di Giacinto \\ Consiglio per la ricerca in agricoltura e l'analisi dell' economia agraria, CREA-OLI Olive Growing and Oil \\ Industry Research Centre, Viale Petruzzi 75, 65013 Città Sant’Angelo (PE). Italy
}

(Received: 26 June 2014; accepted: 26 August 2014)

\begin{abstract}
The quality of monovarietal extra virgin olive oil from cv. 'Taggiasca' is influenced by many factors that have impact on shelf-life as well as on sensory and healthy properties of the product. The aim of the work was to recreate the conditions similar to those in consumer sales point (conditions of "shelf"), maintaining the olive oil packaged in dark-green bottles at room temperature (between 18 and $25^{\circ} \mathrm{C}$ ) under artificial light and away from heat sources, monitoring the oils up to 12 months from bottling with quarterly sampling for the main chemical, physico-chemical, and sensory parameters related to the quality. After one year of storage, an organoleptic alteration with reduction of the attributes 'fruity', 'pungent', and 'bitter', as well as the occurrence of 'rancid' defect, was observed. This alteration was found to be accompanied by a decrease in phenolic substances and tocopherols and an increase in primary and secondary oxidation products. The composition of the volatile fraction showed a slight increase of substances related to rancid defect, a constant trend of compounds related to fruitiness, and a slight decrease in alcohols. It can be concluded that the optimum time of storage of the oil under the above-mentioned conditions is approximately 9 months.
\end{abstract}

Keywords: monovarietal extra virgin olive oil, Olea europaea, rancid defect, shelf-life

Lipid hydrolysis and oxidation are the main causes of the extra virgin olive oil (EVOO) quality deterioration and its reaction rate determines the shelf-life of this product. These processes lead to the formation of off-flavours along with a decrease in nutritional property (ANGEROSA et al., 2004). EVOO is a source of natural antioxidants, such as tocopherols, chlorophyll, and carotenoid pigments, and biophenols, involved in different mechanisms providing an effective defence against free radicals (SERVILI et al., 2014). The antioxidant content depends on many factors, in particular on genetic profile of Olea europaea cultivars. The study of the chemical and physico-chemical characteristics of monovarietal extra virgin olive oil (MEVOO) is useful in order to establish the storage time to preserve as long as possible the organoleptic and nutritional quality within the limits established for the extravirgin category declared by the European Union Commission Regulation EEC (1991) and its subsequent modifications.

The oxidative alterations that occur during the conservation of oil have been widely studied (Gomez-Alonso et al., 2007; SAmAniEgo-SÁnchez et al., 2012). The influence of temperature, light, metal contents, the presence of pigments and antioxidants, and oxygen partial pressure on the oxidative process was also studied (Gutierrez \& Fernandez, 2002; Stefanoudaki et al., 2010; Ayton et al., 2012; Nabil et al., 2012). However, only few works have been carried out to study the shelf-life of monovarietal extra virgin olive oils (LANZA et

\footnotetext{
* To whom correspondence should be addressed

Phone: +39-085-95294; fax: +39-085-959518; e-mail: barbara.lanza@entecra.it
} 
al., 2013b). Several studies demonstrated that the storage conditions, such as containers, temperature, exposure to light and air, influence the packaged oils (ANGEROSA et al., 1993; Morello et al., 2004; MÉndez \& Falqué, 2007; Romani et al., 2007). The purpose of the present work was to reproduce conditions similar to those referred to in sales point (conditions of "shelf"), maintaining the olive oil packaged in dark-green bottles at room temperature (between 18 and $25^{\circ} \mathrm{C}$ ) under artificial light and away from heat sources, monitoring the oils up to 12 months from bottling with quarterly sampling for the main chemical, physicochemical, and sensory parameters related to the quality.

\section{Materials and methods}

\subsection{Materials and storage conditions}

Monovarietal extra virgin olive oils (MEVOO) obtained from Olea europaea L. cv 'Taggiasca' were used in this study. The oils were extracted in industrial mill by three-phase continuous system and then packaged in full filled 11 bottles of dark-green glass. The bottles were maintained at room temperature (between 18 and $25^{\circ} \mathrm{C}$ ) under artificial light and away from heat sources. The monitoring was performed analysing the oils up to 12 months from bottling with quarterly sampling (EV1 at 0 time, EV2 after three months of storage, EV3 after six months of storage, EV4 after nine months of storage, and EV5 after 12 months of storage).

\subsection{Methods}

Several analyses were regularly performed to determine and quantify any possible evolution of parameters related to the olive oil quality.

1.2.1. Free acidity, peroxide index, and spectrophotometric investigation in the ultraviolet. Free fatty acid content (percent oleic acid), peroxide index (PI expressed as milliequivalents active oxygen per $\mathrm{kg}$ oil ( meq $_{2}$ per $\left.\mathrm{kg}\right)$ ), and $\mathrm{UV}$ absorption characteristics $\left(\mathrm{K}_{232}, \mathrm{~K}_{270}, \Delta \mathrm{K}\right)$ were determined in duplicate according to the analytical methods described in EEC (1991) and its subsequent modifications.

1.2.2. Pigments, tocopherols, and biophenols. The determination of pigment (chlorophylls and carotenoids), tocopherol, and biophenol contents were performed according to the methods described in a previous work (LANZA et al., 2013a).

1.2.3. Head-space volatile compounds. The volatile compounds were stripped from 50 g oil samples with $\mathrm{N}_{2}\left(1.2 \mathrm{dm}^{3} \mathrm{~min}^{-1}\right.$, for $2 \mathrm{~h}$ at $\left.37^{\circ} \mathrm{C}\right)$ using $10 \mathrm{mg}$ 1-nonanol added as the internal standard. The samples were adsorbed onto $50 \mathrm{mg}$ activated charcoal and eluted with $1 \mathrm{ml}$ diethyl ether. Quantitative analysis was carried out using gas chromatography, with a Carlo Erba (Milan, Italy) Mega Series 5160 gas chromatograph equipped with a Restek STABIL-WAX silica capillary column (length $60 \mathrm{~m}$; i.d. $0.32 \mathrm{~mm}$; film thickness $0.5 \mu \mathrm{m}$ ), an on-column injection system, a $\mathrm{CO}_{2}$ cryogenic accessory to hold the oven at $28^{\circ} \mathrm{C}$, and flame ionisation detector set at $230^{\circ} \mathrm{C}$. The oven temperature programme was run at $28{ }^{\circ} \mathrm{C}$ for 6 min, increasing at $1.0^{\circ} \mathrm{C} \mathrm{min}^{-1}$ to $33{ }^{\circ} \mathrm{C}$ (no hold), then $1.8^{\circ} \mathrm{C} \mathrm{min}^{-1}$ to $110{ }^{\circ} \mathrm{C}$ (no hold), and then $2.5^{\circ} \mathrm{C} \mathrm{min}^{-1}$ to $215^{\circ} \mathrm{C}$, where it was held for $10 \mathrm{~min}$. The carrier gas was $\mathrm{H}_{2}$ at $40 \mathrm{kPa}$. The injection volume was $0.5 \mu \mathrm{l}$. Quantification was performed by peak area integration with a Carlo Erba Mega Series integrator. Concentrations are expressed as $\mathrm{mg} \mathrm{kg}^{-1}$ 1-nonanol. 
1.2.4. Sensory analysis. The evaluation of the monovarietal olive oils was performed under the conditions described in EEC (1991) and its subsequent modifications (Annex XII) by the CREA-OLI Panel recognized by the International Olive Oil Council (IOC) and the Italian Ministry of Agricultural, Food and Forestry Policies (MIPAAF). Each taster of the panel smelled and tasted the oil sample, in order to analyse the olfactory and gustatory perceptions according to the devised profile sheet of the Annex XII. The attributes evaluated were: fusty/muddy sediment, rancid, musty/humid/earthy, winery/vinegary/acid/sour, metallic, fruity (greenly and ripely), bitter, pungent. The profile sheet uses a 10-point intensity scale that ranges from 0 (no perception) to 10 (extreme). All of these analyses were carried out in duplicate for each sample. To elaborate the sensory data, the method for calculating the median and the confidence intervals was used as detailed in Annex XII, taking into account the attributes with a robust coefficient of variation of $20 \%$ or less.

1.2.5. Data analysis and statistics. Chemical data were reported as mean values of two replications. Data were subjected to one-way ANOVA and the differences were compared with Fisher's test at 0.05 probability level.

\section{Results and discussion}

The evolution of the main chemical and physico-chemical characteristics of the oil during the storage period are reported in Table 1.

The acidity shows a very slight increase, probably due to the release of fatty acids from lipids by hydrolysis.

The MEVOO before the storage had a considerable content of natural antioxidants such as $\alpha$-tocopherol $\left(168 \mathrm{mg} \mathrm{kg}^{-1}\right)$. After 3 months of storage, the $\alpha$-tocopherol content decreased consistently $\left(118 \mathrm{mg} \mathrm{kg}^{-1}\right)$, remained constant until the 9th month of storage, then decreased reaching the value of $64 \mathrm{mg} \mathrm{kg}^{-1}$ at the end of the 12 months of storage.

Table 1. Chemical and physico-chemical characteristics of MEVOO during storage

\begin{tabular}{|c|c|c|c|c|c|}
\hline Parameters & EV1 & EV2 & EV3 & EV4 & EV5 \\
\hline Free fatty acidity (g oleic acid/100 g) & $0.29^{\mathrm{a}}$ & $0.32^{\mathrm{a}}$ & $0.33^{\mathrm{a}}$ & $0.31^{\mathrm{a}}$ & $0.35^{\mathrm{a}}$ \\
\hline $\begin{array}{l}\text { Peroxide value ( } \mathrm{mEq} \mathrm{O}_{2} \mathrm{~kg}^{-1} \text { ) } \\
\text { UV spectrophotometry }\end{array}$ & $11.9^{\mathrm{a}}$ & $18.6^{\mathrm{b}}$ & $17.3^{b}$ & $11.0^{\mathrm{a}}$ & $12.8^{\mathrm{a}}$ \\
\hline $\mathrm{K}_{232}$ & $1.970^{\mathrm{a}}$ & $1.934^{\mathrm{a}}$ & $1.928^{\mathrm{a}}$ & $1.984^{\mathrm{a}}$ & $2.145^{b}$ \\
\hline $\mathrm{K}_{270}$ & $0.111^{\mathrm{a}}$ & $0.109^{\mathrm{a}}$ & $0.108^{\mathrm{a}}$ & $0.129^{a}$ & $0.132^{\mathrm{a}}$ \\
\hline$\Delta \mathrm{K}$ & $-0.001^{\mathrm{a}}$ & $0.002^{\mathrm{a}}$ & $0.003^{\mathrm{a}}$ & $0.004^{\mathrm{a}}$ & $0.003^{\mathrm{a}}$ \\
\hline \multicolumn{6}{|l|}{ Carotenoids (VIS spectrophotometry) } \\
\hline $\mathrm{A}_{414}$ & $1.043^{\mathrm{a}}$ & $1.026^{\mathrm{a}}$ & $1.036^{\mathrm{a}}$ & $1.026^{\mathrm{a}}$ & $1.009^{\mathrm{a}}$ \\
\hline $\mathrm{A}_{450}$ & $0.804^{\mathrm{a}}$ & $0.803^{\mathrm{a}}$ & $0.805^{\mathrm{a}}$ & $0.799^{\mathrm{a}}$ & $0.770^{\mathrm{a}}$ \\
\hline $\mathrm{A}_{477}$ & $0.636^{\mathrm{a}}$ & $0.645^{\mathrm{a}}$ & $0.644^{\mathrm{a}}$ & $0.627^{\mathrm{a}}$ & $0.610^{\mathrm{a}}$ \\
\hline Chlorophyll ( $\left.\mathrm{mg} \mathrm{kg}^{-1}\right)$ & $5^{\mathrm{a}}$ & $5^{\mathrm{a}}$ & $5^{\mathrm{a}}$ & $5^{\mathrm{a}}$ & $5^{\mathrm{a}}$ \\
\hline$\alpha$-Tocopherol $\left(\mathrm{mg} \mathrm{kg}^{-1}\right)$ & $168^{a}$ & $118^{b}$ & $114^{b}$ & $119^{b}$ & $64^{c}$ \\
\hline$\gamma$-Tocopherol $\left(\mathrm{mg} \mathrm{kg}^{-1}\right)$ & $6^{\mathrm{a}}$ & $3^{a}$ & $5^{\mathrm{a}}$ & $5^{\mathrm{a}}$ & $4^{\mathrm{a}}$ \\
\hline
\end{tabular}

Mean values within the same row followed by common superscript letters do not differ significantly $(\mathrm{P}<0.05)$ 
The peroxide value increased up to a maximum of 19 (reached at 3 months of storage) and then started to decrease. This behaviour can be explained by an initial increase in hydroperoxides, which are flavourless compounds produced during the primary step of oxidation. Subsequently these compounds give rise to substances responsible for off-flavours (secondary oxidation) (FrANKEL, 1980).

$\mathrm{K}_{232}$ coefficient remained nearly constant or slightly increased after 9 months of storage but has not exceeded the limit of 2.50 fixed for the "extra" quality (EV5=2.145). The $\mathrm{K}_{270}$ coefficient remained unaltered throughout the rest of the storage period.

Also, pigment (chlorophyll and carotenoid) content remained practically constant during all the storage. The dark-green glass protects chlorophylls from oxidative degradation, absorbing part of the radiation at wavelengths corresponding to their absorption, so that reduces the activity of the initiators of the reaction of hydroperoxide formation, and limits the deterioration of the quality of the oil.

Table 2. Evolution of phenolic compounds of MEVOO during storage.

\begin{tabular}{|c|c|c|c|c|c|}
\hline Phenolic compounds & EV1 & EV2 & EV3 & EV4 & EV5 \\
\hline 3,4-DHPEA (Hydroxytyrosol) & $3.73^{\mathrm{a}}$ & $5.07^{b}$ & $5.67^{\mathrm{b}}$ & $4.84^{b}$ & $5.21^{\mathrm{b}}$ \\
\hline$p$-HPEA (Tyrosol) & $5.18^{\mathrm{a}}$ & $6.56^{\mathrm{b}}$ & $7.30^{\mathrm{b}}$ & $6.84^{b}$ & $6.86^{b}$ \\
\hline Vanillic acid & $0.52^{\mathrm{a}}$ & $0.64^{\mathrm{a}}$ & $0.59^{\mathrm{a}}$ & $0.59^{\mathrm{a}}$ & $0.43^{\mathrm{a}}$ \\
\hline Vanillin & $0.11^{\mathrm{a}}$ & $1.36^{\mathrm{b}}$ & $1.08^{\mathrm{b}}$ & $1.25^{\mathrm{b}}$ & $0.47^{\mathrm{c}}$ \\
\hline$p$-Coumaric acid & $0.00^{\mathrm{a}}$ & $0.49^{b}$ & $0.48^{\mathrm{b}}$ & $0.23^{\mathrm{c}}$ & $0.23^{\mathrm{c}}$ \\
\hline Hydroxytyrosyl acetate & $2.49^{\mathrm{a}}$ & $2.61^{\mathrm{a}}$ & $2.85^{\mathrm{a}}$ & $0.48^{b}$ & $0.35^{b}$ \\
\hline Ferulic acid & $0.00^{\mathrm{a}}$ & $0.32^{b}$ & $0.14^{\mathrm{a}}$ & $2.46^{\mathrm{c}}$ & $2.04^{\mathrm{c}}$ \\
\hline$o$-Coumaric acid & $0.31^{\mathrm{a}}$ & $0.26^{\mathrm{a}}$ & $0.28^{\mathrm{a}}$ & $0.26^{\mathrm{a}}$ & $0.24^{\mathrm{a}}$ \\
\hline 3,4-DHPEA-EDA ox & $0.71^{\mathrm{a}}$ & $1.49^{\mathrm{b}}$ & $2.11^{\mathrm{c}}$ & $2.26^{\mathrm{c}}$ & $4.61^{\mathrm{d}}$ \\
\hline 3,4-DHPEA-EDA & $10.39^{\mathrm{a}}$ & $7.69^{b}$ & $8.07^{\mathrm{b}}$ & $6.60^{\mathrm{c}}$ & $6.44^{\mathrm{c}}$ \\
\hline Oleuropein & $2.76^{\mathrm{a}}$ & $4.01^{b}$ & $1.88^{\mathrm{c}}$ & $1.66^{\mathrm{c}}$ & $1.08^{\mathrm{d}}$ \\
\hline 3,4-DHPEA-EA (Oleuropein aglycon) & $0.54^{\mathrm{a}}$ & $0.42^{\mathrm{a}}$ & $0.53^{\mathrm{a}}$ & $0.34^{\mathrm{a}}$ & $0.52^{\mathrm{a}}$ \\
\hline Tyrosyl acetate & $0.41^{\mathrm{a}}$ & $1.38^{\mathrm{b}}$ & $2.22^{\mathrm{c}}$ & $0.77^{\mathrm{d}}$ & $0.45^{\mathrm{a}}$ \\
\hline$p$-HPEA-EDA ox & $3.56^{\mathrm{a}}$ & $3.71^{\mathrm{a}}$ & $7.35^{\mathrm{b}}$ & $8.00^{b}$ & $11.10^{\mathrm{c}}$ \\
\hline p-HPEA-EDA (Oleocanthal) & $24.01^{\mathrm{a}}$ & $13.64^{b}$ & $12.57^{b}$ & $6.70^{\mathrm{c}}$ & $6.93^{c}$ \\
\hline Lignans & $41.15^{\mathrm{a}}$ & $50.75^{b}$ & $55.89^{b}$ & $49.38^{b}$ & $31.40^{\mathrm{c}}$ \\
\hline Cinnamic acid & $0.75^{\mathrm{a}}$ & $0.74^{\mathrm{a}}$ & $1.94^{\mathrm{b}}$ & $0.74^{\mathrm{a}}$ & $0.99^{\mathrm{a}}$ \\
\hline p-HPEA-EA (Ligstroside aglycon) & $0.64^{\mathrm{a}}$ & $0.59^{\mathrm{a}}$ & $1.39^{\mathrm{b}}$ & $1.57^{\mathrm{b}}$ & $1.78^{\mathrm{c}}$ \\
\hline 3,4-DHPEA, -EA, H ox & $0.00^{\mathrm{a}}$ & $0.41^{\mathrm{b}}$ & $0.88^{\mathrm{c}}$ & $1.00^{\mathrm{c}}$ & $5.08^{\mathrm{d}}$ \\
\hline 3,4-DHPEA, -EA, H & $14.34^{\mathrm{a}}$ & $12.02^{b}$ & $10.87^{\mathrm{c}}$ & $5.36^{\mathrm{d}}$ & $5.60^{\mathrm{d}}$ \\
\hline$p$-HPEA, -EA, H ox & $6.30^{\mathrm{a}}$ & $11.46^{\mathrm{b}}$ & $13.30^{\mathrm{c}}$ & $7.85^{\mathrm{d}}$ & $8.70^{\mathrm{d}}$ \\
\hline Apigenin & $0.61^{\mathrm{a}}$ & $0.71^{\mathrm{a}}$ & $0.76^{\mathrm{a}}$ & $0.76^{\mathrm{a}}$ & $0.57^{\mathrm{a}}$ \\
\hline Methyl-luteolin & $0.66^{\mathrm{a}}$ & $0.22^{b}$ & $0.36^{\mathrm{b}}$ & $4.63^{c}$ & $4.99^{c}$ \\
\hline$p$-HPEA, -EA, H & $3.34^{\mathrm{a}}$ & $5.73^{\mathrm{b}}$ & $5.41^{\mathrm{b}}$ & $2.17^{\mathrm{c}}$ & $1.38^{\mathrm{d}}$ \\
\hline
\end{tabular}

Results are expressed as $\mathrm{mg} \mathrm{kg}^{-1}$ of tyrosol

Mean values within the same row followed by common superscript letters do not differ significantly $(\mathrm{P}<0.05)$

During storage, the quantity of main active biophenols (secoiridoids and lignans) of virgin olive oil decreases (Table 2). Secoiridoids developing during crushing from the 
hydrolysis of oleuropein and ligstroside include an isomer of the oleuropein aglycon (3,4-DHPEA-EA), the ligstroside aglycon ( $p$-HPEA-EA) and the dialdehydic form of decarboxymethyl elenolic acid linked to hydroxytyrosol (3,4-DHPEA) and tyrosol ( $p$-HPEA), respectively 3,4-DHPEA-EDA and $p$-HPEA-EDA or oleocanthal. The main lignans found in virgin olive oil are $(+)$-1-acetoxypinoresinol and (+)-1-pinoresinol. Both secoiridoids and lignans affect the quality of the sensory and health properties of virgin olive oil: 3,4-DHPEAEA, $p$-HPEA-EA, and, with a minor role, 3,4-DHPEA-EDA are responsible of 'bitter' sensation, while $p$-HPEA-EDA (oleocanthal) determines 'pungent' sensation (Esti et al., 2009). During storage in the tanks, the phenolic composition of extra virgin olive oil is modified by the endogenous enzymatic activities contained in the cloudy phase. Oil filtration partially removes the water and enzymes from extra virgin olive oil, and enables to stabilize the phenolic content during its storage. Simple biophenols, such as $p$-HPEA and 3,4-DHPEA, increase probably due to the aglycon degradation (Table 2). Also, oxidised forms of aglycons increase (Table 2): phenols fight oxidation giving electrons to free radicals and oxidising themselves.

The olive oil aromatic profile changes during storage, due to the neo-formation of volatile compounds responsible for common defect referred to as "rancid" (pentanal, heptanal, and nonanal; Table 3). This runs parallel to the increase in saturated aldehyde hexanal content (from 1.86 to $2.96 \mathrm{mg} \mathrm{kg}^{-1}$; Table 3 ) in the oxidation process, that in this case could be considered a useful marker of oxidation, since it comes only from the secondary oxidation of the linoleic hydroperoxide radical (SolinAs et al., 1987). The composition of the volatile fraction shows, in addition to a slight increase of substances related to rancid defect, a slight decrease in compounds related to fruitiness (cis-3-hexenal, 1-hexanol and cis-3hexen-1-ol; Table 3).

Table 3. Evolution of volatile compounds of MEVOO during storage.

\begin{tabular}{|c|c|c|c|c|c|}
\hline Volatile compounds & EV1 & EV2 & EV3 & EV4 & EV5 \\
\hline \multicolumn{6}{|l|}{ Saturated aldehydes } \\
\hline 2-methyl butanal & $0.12^{\mathrm{a}}$ & $0.22^{\mathrm{a}}$ & $0.19^{\mathrm{a}}$ & $0.23^{\mathrm{a}}$ & $0.18^{\mathrm{a}}$ \\
\hline 3-methyl butanal & $0.32^{\mathrm{a}}$ & $0.41^{\mathrm{a}}$ & $0.05^{b}$ & $0.05^{\mathrm{b}}$ & $0.06^{\mathrm{b}}$ \\
\hline Pentanal & $0.00^{\mathrm{a}}$ & $0.00^{\mathrm{a}}$ & $0.00^{\mathrm{a}}$ & $0.00^{\mathrm{a}}$ & $0.09^{b}$ \\
\hline Hexanal & $1.86^{\mathrm{a}}$ & $2.69^{b}$ & $2.05^{b}$ & $2.74^{b}$ & $2.96^{\mathrm{b}}$ \\
\hline Heptanal & $0.00^{\mathrm{a}}$ & $0.07^{\mathrm{b}}$ & $0.06^{\mathrm{b}}$ & $0.06^{\mathrm{b}}$ & $0.06^{b}$ \\
\hline Octanal & $0.01^{\mathrm{a}}$ & $0.01^{\mathrm{a}}$ & $0.03^{\mathrm{a}}$ & $0.07^{\mathrm{a}}$ & $0.04^{\mathrm{a}}$ \\
\hline Nonanal & $0.00^{\mathrm{a}}$ & $0.00^{\mathrm{a}}$ & $0.03^{\mathrm{a}}$ & $0.02^{\mathrm{a}}$ & $0.13^{b}$ \\
\hline \multicolumn{6}{|l|}{$\underline{\text { Unsaturated aldehydes }}$} \\
\hline trans-2-pentenal & $0.01^{\mathrm{a}}$ & $0.03^{\mathrm{a}}$ & $0.00^{\mathrm{a}}$ & $0.00^{\mathrm{a}}$ & $0.00^{\mathrm{a}}$ \\
\hline cis-3-hexenal & $0.16^{\mathrm{a}}$ & $0.15^{\mathrm{a}}$ & $0.11^{\mathrm{b}}$ & $0.11^{\mathrm{b}}$ & $0.07^{\mathrm{c}}$ \\
\hline trans-2-hexenal & $30.12^{\mathrm{a}}$ & $36.30^{\mathrm{a}}$ & $33.57^{\mathrm{a}}$ & $33.92^{\mathrm{a}}$ & $31.92^{\mathrm{a}}$ \\
\hline trans-2-heptenal & $0.03^{\mathrm{a}}$ & $0.03^{\mathrm{a}}$ & $0.02^{\mathrm{a}}$ & $0.03^{\mathrm{a}}$ & $0.02^{\mathrm{a}}$ \\
\hline trans-2-octenal & $0.00^{\mathrm{a}}$ & $0.00^{\mathrm{a}}$ & $0.00^{\mathrm{a}}$ & $0.00^{\mathrm{a}}$ & $0.01^{\mathrm{a}}$ \\
\hline \multicolumn{6}{|l|}{$\underline{\text { Saturated alcohols }}$} \\
\hline Ethanol & $13.66^{\mathrm{a}}$ & $17.34^{b}$ & $13.04^{\mathrm{a}}$ & $17.23^{b}$ & $12.88^{\mathrm{a}}$ \\
\hline 1-propanol & $0.13^{\mathrm{a}}$ & $0.08^{\mathrm{a}}$ & $0.07^{\mathrm{a}}$ & $0.10^{\mathrm{a}}$ & $0.04^{b}$ \\
\hline
\end{tabular}


Table 3 continued

\begin{tabular}{|c|c|c|c|c|c|}
\hline Volatile compounds & EV1 & EV2 & EV3 & EV4 & EV5 \\
\hline Iso-propanol & $0.04^{\mathrm{a}}$ & $0.05^{\mathrm{a}}$ & $0.20^{\mathrm{b}}$ & $0.12^{b}$ & $0.24^{\mathrm{b}}$ \\
\hline 2-Butanol & $0.01^{\mathrm{a}}$ & $0.03^{\mathrm{a}}$ & $0.03^{\mathrm{a}}$ & $0.02^{\mathrm{a}}$ & $0.03^{\mathrm{a}}$ \\
\hline Iso-butanol & $0.28^{\mathrm{a}}$ & $0.34^{\mathrm{a}}$ & $0.28^{\mathrm{a}}$ & $0.31^{\mathrm{a}}$ & $0.27^{\mathrm{a}}$ \\
\hline 2-methyl-3-butanol & $0.00^{\mathrm{a}}$ & $0.00^{\mathrm{a}}$ & $0.00^{\mathrm{a}}$ & $0.00^{\mathrm{a}}$ & $0.01^{\mathrm{a}}$ \\
\hline 3-methyl-1-butanol & $0.93^{\mathrm{a}}$ & $1.04^{\mathrm{a}}$ & $0.86^{\mathrm{a}}$ & $0.94^{\mathrm{a}}$ & $0.83^{\mathrm{a}}$ \\
\hline 3-pentanol & $0.07^{\mathrm{a}}$ & $0.09^{\mathrm{a}}$ & $0.02^{\mathrm{a}}$ & $0.01^{\mathrm{a}}$ & $0.02^{\mathrm{a}}$ \\
\hline 1-pentanol & $0.14^{\mathrm{a}}$ & $0.15^{\mathrm{a}}$ & $0.12^{\mathrm{a}}$ & $0.13^{\mathrm{a}}$ & $0.14^{\mathrm{a}}$ \\
\hline 2-methyl-2-pentanol & $0.00^{\mathrm{a}}$ & $0.03^{\mathrm{a}}$ & $0.09^{\mathrm{b}}$ & $0.09^{b}$ & $0.09^{\mathrm{b}}$ \\
\hline 1-hexanol & $2.40^{\mathrm{a}}$ & $2.36^{\mathrm{a}}$ & $2.11^{b}$ & $2.20^{\mathrm{b}}$ & $1.98^{\mathrm{b}}$ \\
\hline 1-heptanol & $0.02^{\mathrm{a}}$ & $0.01^{\mathrm{a}}$ & $0.01^{\mathrm{a}}$ & $0.01^{\mathrm{a}}$ & $0.01^{\mathrm{a}}$ \\
\hline \multicolumn{6}{|l|}{ Unsaturated alcohols } \\
\hline 1-penten-3-ol & $1.20^{\mathrm{a}}$ & $1.47^{\mathrm{a}}$ & $1.29^{\mathrm{a}}$ & $1.46^{\mathrm{a}}$ & $1.36^{\mathrm{a}}$ \\
\hline cis-2-penten-1-ol & $0.65^{\mathrm{a}}$ & $0.65^{\mathrm{a}}$ & $0.65^{\mathrm{a}}$ & $0.70^{\mathrm{a}}$ & $0.67^{\mathrm{a}}$ \\
\hline trans-2-penten-1-ol & $0.88^{\mathrm{a}}$ & $0.89^{\mathrm{a}}$ & $0.81^{\mathrm{a}}$ & $0.85^{\mathrm{a}}$ & $0.79^{\mathrm{a}}$ \\
\hline cis-2-hexen-1-ol & $0.02^{\mathrm{a}}$ & $0.02^{\mathrm{a}}$ & $0.02^{\mathrm{a}}$ & $0.02^{\mathrm{a}}$ & $0.01^{\mathrm{a}}$ \\
\hline trans-2-hexen-1-ol & $5.62^{\mathrm{a}}$ & $5.65^{\mathrm{a}}$ & $5.14^{\mathrm{a}}$ & $5.38^{\mathrm{a}}$ & $4.84^{b}$ \\
\hline cis-3-hexen-1-ol & $1.62^{\mathrm{a}}$ & $1.64^{\mathrm{a}}$ & $1.45^{\mathrm{b}}$ & $1.54^{\mathrm{b}}$ & $1.40^{\mathrm{b}}$ \\
\hline trans-3-hexen-1-ol & $0.07^{\mathrm{a}}$ & $0.07^{\mathrm{a}}$ & $0.06^{\mathrm{a}}$ & $0.07^{\mathrm{a}}$ & $0.06^{\mathrm{a}}$ \\
\hline cis-1-octen-3-ol & $0.02^{\mathrm{a}}$ & $0.02^{\mathrm{a}}$ & $0.02^{\mathrm{a}}$ & $0.02^{\mathrm{a}}$ & $0.03^{\mathrm{a}}$ \\
\hline \multicolumn{6}{|l|}{ Esters } \\
\hline Ethyl acetate & $3.09^{\mathrm{a}}$ & $3.82^{b}$ & $3.15^{\mathrm{a}}$ & $4.30^{\mathrm{b}}$ & $3.98^{b}$ \\
\hline Isobutyl acetate & $0.00^{\mathrm{a}}$ & $0.00^{\mathrm{a}}$ & $0.01^{\mathrm{a}}$ & $0.02^{\mathrm{a}}$ & $0.01^{\mathrm{a}}$ \\
\hline Ethyl isobutyrate & $0.00^{\mathrm{a}}$ & $0.00^{\mathrm{a}}$ & $0.13^{b}$ & $0.13^{b}$ & $0.13^{b}$ \\
\hline 1-methyl butyrate & $0.07^{\mathrm{a}}$ & $0.08^{\mathrm{a}}$ & $0.13^{\mathrm{a}}$ & $0.12^{\mathrm{a}}$ & $0.08^{\mathrm{a}}$ \\
\hline 2-methyl butyrate & $0.00^{\mathrm{a}}$ & $0.00^{\mathrm{a}}$ & $0.19^{b}$ & $0.18^{\mathrm{b}}$ & $0.15^{b}$ \\
\hline Hexyl acetate & $0.01^{\mathrm{a}}$ & $0.00^{\mathrm{a}}$ & $0.00^{\mathrm{a}}$ & $0.00^{\mathrm{a}}$ & $0.00^{\mathrm{a}}$ \\
\hline cis-3-hexenyl acetate & $0.11^{\mathrm{a}}$ & $0.13^{\mathrm{a}}$ & $0.10^{\mathrm{a}}$ & $0.11^{\mathrm{a}}$ & $0.11^{\mathrm{a}}$ \\
\hline Ethyl pentanoate & $0.05^{\mathrm{a}}$ & $0.05^{\mathrm{a}}$ & $0.04^{\mathrm{a}}$ & $0.05^{\mathrm{a}}$ & $0.05^{\mathrm{a}}$ \\
\hline \multicolumn{6}{|l|}{$\underline{\text { Saturated ketones }}$} \\
\hline 2-butanone & $0.18^{\mathrm{a}}$ & $0.27^{\mathrm{b}}$ & $0.41^{\mathrm{c}}$ & $0.43^{\mathrm{c}}$ & $0.37^{\mathrm{c}}$ \\
\hline 2-pentanone & $1.81^{\mathrm{a}}$ & $2.32^{b}$ & $2.02^{\mathrm{a}}$ & $2.35^{\mathrm{b}}$ & $2.66^{\mathrm{c}}$ \\
\hline 3-hexanone & $0.12^{\mathrm{a}}$ & $0.15^{\mathrm{a}}$ & $0.13^{\mathrm{a}}$ & $0.11^{\mathrm{a}}$ & $0.13^{\mathrm{a}}$ \\
\hline 2-heptanone & $0.08^{\mathrm{a}}$ & $0.02^{b}$ & $0.03^{b}$ & $0.04^{b}$ & $0.02^{b}$ \\
\hline 2-octanone & $0.41^{\mathrm{a}}$ & $0.41^{\mathrm{a}}$ & $0.36^{\mathrm{a}}$ & $0.39^{\mathrm{a}}$ & $0.36^{\mathrm{a}}$ \\
\hline \multicolumn{6}{|l|}{ Unsaturated ketones } \\
\hline 2-ethylfuran & $0.17^{\mathrm{a}}$ & $0.23^{\mathrm{a}}$ & $0.04^{b}$ & $0.03^{\mathrm{b}}$ & $0.07^{\mathrm{b}}$ \\
\hline 2-pentylfuran & $0.00^{\mathrm{a}}$ & $0.00^{\mathrm{a}}$ & $0.02^{\mathrm{a}}$ & $0.01^{\mathrm{a}}$ & $0.03^{\mathrm{a}}$ \\
\hline 1-penten-3-one & $0.40^{\mathrm{a}}$ & $0.56^{\mathrm{b}}$ & $0.46^{\mathrm{a}}$ & $0.48^{\mathrm{a}}$ & $0.40^{\mathrm{a}}$ \\
\hline 6-methyl-5-hepten-2-one & $0.02^{\mathrm{a}}$ & $0.02^{\mathrm{a}}$ & $0.02^{\mathrm{a}}$ & $0.02^{\mathrm{a}}$ & $0.03^{\mathrm{a}}$ \\
\hline
\end{tabular}


Table 3 continued

\begin{tabular}{llllll}
\hline Volatile compounds & EV1 & EV2 & EV3 & EV4 & EV5 \\
\hline Aliphatic hydrocarbons & & & & & \\
$n$-octane & $1.01^{\mathrm{a}}$ & $1.28^{\mathrm{b}}$ & $1.14^{\mathrm{b}}$ & $1.30^{\mathrm{b}}$ & $1.25^{\mathrm{b}}$ \\
$n$-nonane & $0.03^{\mathrm{a}}$ & $0.05^{\mathrm{a}}$ & $0.19^{\mathrm{b}}$ & $0.41^{\mathrm{c}}$ & $0.24^{\mathrm{b}}$ \\
1-octene & $0.13^{\mathrm{a}}$ & $0.16^{\mathrm{a}}$ & $0.04^{\mathrm{b}}$ & $0.07^{\mathrm{b}}$ & $0.04^{\mathrm{b}}$ \\
2-octene & $0.00^{\mathrm{a}}$ & $0.03^{\mathrm{a}}$ & $0.08^{\mathrm{b}}$ & $0.09^{\mathrm{b}}$ & $0.10^{\mathrm{b}}$ \\
3-octene & $0.02^{\mathrm{a}}$ & $0.08^{\mathrm{b}}$ & $0.01^{\mathrm{a}}$ & $0.00^{\mathrm{a}}$ & $0.01^{\mathrm{a}}$ \\
Pentene dimers & $2.71^{\mathrm{a}}$ & $2.88^{\mathrm{a}}$ & $2.39^{\mathrm{b}}$ & $2.40^{\mathrm{b}}$ & $2.23^{\mathrm{b}}$ \\
a-pinene & $0.80^{\mathrm{a}}$ & $0.86^{\mathrm{a}}$ & $0.79^{\mathrm{a}}$ & $0.84^{\mathrm{a}}$ & $0.77^{\mathrm{a}}$ \\
& & & & & \\
Aromatic hydrocarbons & & & & & \\
p-xylene & $0.09^{\mathrm{a}}$ & $0.13^{\mathrm{b}}$ & $0.15^{\mathrm{b}}$ & $0.15^{\mathrm{b}}$ & $0.17^{\mathrm{b}}$ \\
Carboxylic acids & & & & \\
Acetic acid & $0.01^{\mathrm{a}}$ & $0.19^{\mathrm{b}}$ & $0.34^{\mathrm{c}}$ & $0.20^{\mathrm{b}}$ & $0.12^{\mathrm{b}}$ \\
Propionic acid & $0.03^{\mathrm{a}}$ & $0.02^{\mathrm{a}}$ & $0.02^{\mathrm{a}}$ & $0.02^{\mathrm{a}}$ & $0.02^{\mathrm{a}}$ \\
\hline
\end{tabular}

Results are expressed as $\mathrm{mg} \mathrm{kg}^{-1}$ of 1-nonanol (internal standard).

Mean values within the same row followed by common superscript letters do not differ significantly $(\mathrm{P}<0.05)$

The organoleptic analysis (Table 4) of MEVOO showed the appearance of the rancid defect after 12 months of storage (median of defect=1.5), while fruity, bitter, and pungent properties decreased progressively during storage.

Table 4. Organoleptic analysis of MEVOO during storage

\begin{tabular}{|c|c|c|c|c|c|c|c|c|}
\hline \multirow[t]{2}{*}{ Samplings } & \multicolumn{2}{|c|}{ Fruity } & \multicolumn{2}{|c|}{ Bitter } & \multicolumn{2}{|c|}{ Pungent } & \multicolumn{2}{|c|}{ Defect (rancid) } \\
\hline & Median & $\mathrm{CV} \%$ & Median & $\mathrm{CV} \%$ & Median & $\mathrm{CV} \%$ & Median & $\mathrm{CV} \%$ \\
\hline EV1 & 3.0 & 11.9 & 2.0 & 16.8 & 3.7 & 14.1 & 0.0 & 0.0 \\
\hline EV2 & 3.1 & 14.0 & 2.9 & 9.0 & 3.6 & 8.9 & 0.0 & 0.0 \\
\hline EV3 & 2.8 & 14.3 & 2.1 & 10.4 & 2.9 & 11.0 & 0.0 & 0.0 \\
\hline EV4 & 2.6 & 4.8 & 1.9 & 9.8 & 2.0 & 13.9 & 0.0 & 0,0 \\
\hline EV5 & 2.3 & 13.4 & 1.5 & 7.1 & 2.2 & 6.4 & 1.5 & 9.8 \\
\hline
\end{tabular}

\section{Conclusions}

In conclusion, after one year of storage, an organoleptic alteration reducing fruity, pungent, and bitter attributes, and occurrence of the rancid defect were observed. This alteration is accompanied by a decrease in phenolic substances and tocopherols and an increase in primary and secondary oxidation products. The composition of the volatile fraction showed a slight increase of substances related to rancid defect, a constant trend of compounds related to fruity characteristic, and a slight decrease in alcohols.

The results obtained revealed that the monovarietal extra virgin olive oil studied, packaged in dark-green glass bottles at room temperature (between 18 and $25^{\circ} \mathrm{C}$ ) under artificial light and away from heat sources, with an intermediate initial content of natural antioxidants, has maintained all of its chemical, physical, and sensory properties within the ranges of "Extra virgin olive oil" class during the first nine months of storage. Only at the last 
sampling, to the twelfth month, the product has been downgraded to a "Virgin olive oil" by the sensory evaluation, as a slight defect "rancid" has occurred.

From all of the analyses carried out, it can be concluded that the optimum time of storage of the oil stored as above is up to approximately 9 months.

\section{References}

Angerosa, F., Di Giacinto, L. \& De Mattia, G. (1993): Conservabilità degli oli extra-vergini di oliva confezionati. Nota I (Shelf-life of packaged extra-virgin olive oils. Note I.). Riv. Merceologia, 32, 243-259.

Angerosa, F., Servili, M., Selvaggini, R., Taticchi, A., Esposto, S. \& Montedoro, G.F. (2004): Volatile compounds in virgin olive oil: occurrence and their relationship with the quality. J. Chromatogr. A., 1054, 17-31.

Ayton, J., MaileR, R.J. \& Graham, K. (2012): The effect of storage conditions on extra virgin olive oil quality. Rural Industries Research and Development Corporation, Australian Government. RIRDC Publication No. 12/024. Available from: http://static.oliveoiltimes.com/library/Olive-Oil-Storage-Conditions.pdf.

EEC (1991): Consolidated version of the Commission Regulation EEC No 2568/91 of 11 July 1991 on the characteristics of olive oil and olive-residue oil and on the relevant methods of analysis.

Esti, M., Contini, M., Moneta, E. \& Sinesio, F. (2009): Phenolic compounds and temporal perception of bitterness and pungency in extra-virgin olive oils: Changes occurring throughout storage. Food Chem., 113, 1095-1100.

Frankel, E.N. (1980): Lipid oxidation. Prog. Lipid Res., 19, 1-22.

Gomez-Alonso, S., Mancebo-Campos, V., Desamparados Salvador, M. \& Fregapane, G. (2007): Evolution of major and minor components and oxidation indices of virgin olive oil during 21 months storage at room temperature. Food Chem., 100, 36-42.

Gutierrez, F. \& Fernandez, J.L. (2002): Determinant parameters and components in the storage of virgin olive oil. Prediction of storage time beyond which the oil is no longer of "Extra" quality. J. Agric. Food Chem., 50, 571-577.

Lanza, B., Di Serio, M.G., Giansante, L., Di Loreto, G., Russi, F. \& Di Giacinto, L. (2013a): Effect of pasteurization and storage on quality characteristics of table olives preserved in olive oil. Int. J. Food Sci. Technol., 48, 2630-2637.

Lanza, B., Di Serio, M.G., Giansante, L., Di Loreto, G., Russi, F., Iannucci, E. \& Di Giacinto, L. (2013b): Monitoraggio delle caratteristiche di qualità correlate alla shelf-life di alcuni prodotti liguri ottenuti dalla cv. Taggiasca (olio extra vergine di oliva, olive intere in salamoia, olive denocciolate sott'olio, patè di olive) (Monitoring of quality characteristics related to the shelf-life of some Ligurian olive products obtained from cv 'Taggiasca' (extra virgin olive oil, natural olives in brine, pitted olives in oil, olive paste)). Acta Italus Hort., 10, 306-311.

MÉNDEZ, A.I. \& FALQUÉ, E. (2007): Effect of storage time and container type on the quality of extra-virgin olive oil. Food Control, 18, 521-529.

Morello, J.R., Motilva, M.J., Tovar, M.J. \& Romero, M.P. (2004): Changes in commercial virgin olive oil (cv Arbequina) during storage, with special emphasis on the phenolic fraction. Food Chem., 85, 357-364.

Nabil, B.Y., Youssef, O., Nizar, D., Bechir, B., Chedly, A. \& Mokhtar, Z. (2012): Effect of olive storage period at two different temperatures on oil quality of two Tunisian cultivars of Olea europea, Chemlali and Chétoui. Afr. J. Biotechnol., 11, 888-895.

Romani, A., Lapucci, C., Cantini, C., Ieri, F., Mulinacci, N. \& Visioli, F. (2007): Evolution of minor polar compounds and antioxidant capacity during storage of bottled extra virgin olive oil. J. Agr. Food Chem., 55, $1315-1320$.

Samaniego-SÁnchez, C., Oliveras-López, M.J., Quesada-Granados, J.J., Villalón-Mir, M. \& Serrana, H.L.-G. (2012): Alterations in picual extra virgin olive oils under different storage conditions. Eur. J. Lipid Sci. Tech., 114, 194-204.

Servili, M., Sordini, B., Esposto, S., Urbani, S., Veneziani, G., Di Maio, I., Selvaggini, R. \& Taticchi, A. (2014): Biological activities of phenolic compounds of extra virgin olive oil. Antioxidants, 3, 1-23.

Solinas, M., Angerosa, F. \& Cucurachi, A. (1987): Connessione tra prodotti di neoformazione ossidativa delle sostanze grasse e insorgenza del difetto di rancidità all'esame organolettico. Nota II. Determinazione quantitativa (Connection between products of oxidative neoformation of fatty substances and onset of rancid defect by organoleptic analysis. Note II. Quantitative determination). Riv. Ital. Sostanze Gr., 64, 137-145.

Stefanoudaki, E., Williams, M. \& Harwood, J. (2010): Changes in virgin olive oil characteristics during different storage conditions. Eur. J. Lipid Sci. Tech., 112, 906-914.

Acta Alimentaria 44, 2015 